\title{
Scientific explanation and the Troubles with Causal Explanations in physics
}

Andrés Rivadulla

Universidad Complutense de Madrid, España

(c) $\underset{\mathrm{Br}}{\mathrm{i}}$ 


\title{
Scientific explanation and the Troubles with Causal Explanations in physics'
}

\begin{abstract}
Fifty years ago, Carl Gustav Hempel published his famous book Aspects of Scientific Explanation. Since then the number of publications on this subject has grown exponentially. An occasion like this deserves to be commemorated. In this article I offer a modest tribute to this great methodologist of science.
\end{abstract}

This paper tackles the uses of explanation in theoretical sciences. In particular it is concerned with the possibility of causal explanations in physics. What I intend to do is to focus on the issue of whether the explanation of Kepler's empirical laws of the planetary motions can be a causal explanation. More specifically my point is: can the deductive subsumption of Kepler's $3^{\text {rd }}$ Law (also known as Kepler's 1-2-3 law) under theoretical principles provide a causal explanation for the planetary motions?

My answer is a definitive no. As a matter of fact, on occasion subsumptions occur under differing theoretical principles that are incompatible with one another. In such cases we would have incompatible scientific explanations. This is precisely the situation facing the scientific explanation of Kepler's laws, in particular the third law. Since there exist incompatible gravitational theories, it is impossible for the scientific account of Kepler's law to be a causal explanation of the planetary motions. This is just one example of the difficulties faced by causal explanations in sciences such as theoretical physics.

Keywords: scientific explanation, theoretical explanation, incompatibility, causal explanation, Newtonian mechanics, relativity theory.

\section{Explicación científica y los problemas de las explicaciones causales en física}

\begin{abstract}
Resumen: hace cincuenta años, Carl Hempel publicó su famoso libro Aspects of Scientific Explanation. Desde entonces el número de publicaciones sobre este tema ha crecido exponencialmente. Una ocasión como ésta merece ser conmemorada. Así pues, en este artículo ofrezco un modesto homenaje a este gran metodólogo de la ciencia.
\end{abstract}

El presente trabajo aborda los usos de la explicación en las ciencias teóricas. En particular se ocupa de la posibilidad de explicaciones causales en física. Lo que intento es centrarme en si la explicación de las leyes empíricas de Kepler de los movimientos planetarios puede ser una explicación causal. De modo más específico, mi problema es: iproporciona la subsunción deductiva bajo principios teóricos de la 3a Ley de Kepler (también conocida como ley 1-2-3 de Kepler) una explicación causal de los movimientos planetarios?

Mi respuesta es, definitivamente, no. En efecto, en ocasiones las subsunciones tienen lugar bajo principios teóricos diferentes e incompatibles entre sí. En tales casos tendremos explicaciones científicas incompatibles. Ésta es precisamente la situación a la que se enfrentan las leyes de Kepler, en particular la tercera ley. Como hay teorías gravitacionales incompatibles, es imposible que la explicación científica de la ley de Kepler constituya una explicación causal de los movimientos planetarios. Éste es solo un ejemplo de las dificultades a las que se enfrentan las explicaciones causales en ciencias como la física teórica.

Palabras clave: explicación científica, explicación teórica, incompatibilidad, explicación causal, mecánica newtoniana, teoría de la relatividad.

Fecha de recepción: 1 de noviembre de 2016

Fecha de aceptación: 6 de abril de 2017

Forma de citar (APA): Rivadulla, A. (2017). Scientific explanation and the troubles with causal explanations in physics. Revista Filosofía UIS, 16(2), doi: http://dx.doi.org/10.18273/revfil.v16n2-2017008

Forma de citar (Harvard):Rivadulla, A. (2017). Scientific explanation and the troubles with causal explanations in physics. Revista Filosofía UIS, 16(2), 159-172.

Andrés Rivadulla: español. Doctor en Filosofía y Letras. Profesor de Lógica y Filosofía de la Ciencia, Universidad Complutense de Madrid.

Correo electrónico: arivadulla@filos.ucm.es

* Artículo de investigación científica y tecnológica.

${ }^{1}$ Research Project FFI2014-52224-P financed by the Ministry of Economy and Competitiveness of the Government of the Kingdom of Spain, and Complutense Research Group 930174. I am very grateful to two anonymous referees for their helpful comments on a previous version of this article. 


\section{Scientific explanation and the Troubles with Causal Explanations in physics}

\section{Introduction}

In an interview with the Korean economist Ha-Joon Chang, published in the Spanish newspaper El País on Sunday August 2, 2015, the interviewer Pablo Guimón asks Chang if economics is a science. Part of Chang's answer is: "if you think it is a science, you can not accept that there are two or three explanations of the same thing".

To give a causal explanation of something (the explicandum) means to identify unequivocally its cause(s). Allegedly science can provide clear grounds for events. For instance explanations of why eclipses occur, or why the seasons happen clearly identify in principle the causes of these phenomena. So we say that solar eclipses occur because in its rotation around the Earth, the Moon gets in the visual between Earth and the Sun. The moon casts shadow on Earth and obscures a strip of the same. The Sun sets in broad daylight. Before knowing the astronomical cause of a solar eclipse the number of bizarre 'explanations' that has suffered mankind has been important. To date we know that the seasons follow one another because of both: the tilt of the Earth -not because of the distance from Earth to the Sun- and the annual orbit of our planet around the sun. And like these we could find plenty of causal explanations in science.

Causal explanation is explanation by reference to (efficient) causes: the occurrence of certain events or circumstances that motivates others to happen. We say that the former cause the latter. It is true that David Hume argued that it is logically impossible to decide, beyond any reasonable doubt, that an event causes another. But this position is unreasonable. Nowadays Nancy Cartwright has opposed Hume. For her "causality is an objective feature of our scientific image of nature" (1989, p. 2) and "science needs a separate notion of causal law" (141). This position seems reasonable in principle. 
Since the eighteenth century, facing Hume's scepticism attempts by Jakob Bernoulli, Thomas Bayes and Pierre Simon de Laplace, followed by developments in theoretical statistics in the nineteenth and twentieth centuries -see for example Rivadulla (1991)- were aimed at finding ways to model learning from experience. Or, in other words: to find out causes in databases. Finally, contemporary philosophy of science, rehabilitating the context of scientific discovery, despised by Popper, Reichenbach and in general by all methodologists of science until the seventies, has recovered abduction, inference to the best explanation, a form of reasoning -fallible of course- that allows to proposing the most reasonable among several competing hypotheses as the tentative cause of a phenomenon. Indeed, it is easy to see -on this subject see also Rivadulla (2015) - that from its very beginnings to the present day Western science has used abduction to postulate most interesting hypotheses about the causes of the investigated phenomena. That is, facing the paralyzing position of Hume, which threatened to produce the collapse of scientific practice, or convert it in a by-product of everyday psychology, science and philosophy, walking as almost always hand in hand, offered ingenious and fertile alternatives to the problem of the investigation of causes.

However this is not the issue that I tackle in this paper. Nor is it the aim of this article to restart the discussion -so often taken up in the history of philosophyaround the concept of causality. So, I will not refer the views of Berkeley, Duhem, Poincaré or Mach, even by way of illustration that the causal explanation stumbles in history upon significant detractors. For reasons of economy and simplicity I'm not going either to enter into discussions among different contemporary methodologists of science about the different forms and implications of the problem of scientific explanation. I will not even refer to the problem of determinism from the perspective of orthodox quantum mechanics, since there are other quantum theories that do not question the idea of causality.

The question that interests me is, say, different. And it is implicit in the examples of causal explanation mentioned above. Indeed, these examples occult a problem, since we are assuming as facts both, that the Moon orbits around the Earth -for the explanation of the solar eclipses- and that the Earth orbits, with a given inclination of its axis, around the Sun -for the explanation of the seasons' succession. Briefly: That the Moon is a satellite of the Earth, and that the Earth is a solar planet. But assuming this amounts to accepting that in the context of scientific explanation, a reference to theories seems unavoidable. So any scientific explanation is ultimately a theoretical explanation. And if this is so, then the question of whether any scientific explanation is causal becomes secondary.

Indeed there is no doubt to date that the Moon is a (the) satellite of the Earth, and that the Earth is a planet of the Sun. Pictures taken from artificial satellites situated conveniently far away confirm this. But until recently only the accumulation of observations and theoretical calculations combined with each 
other was what allowed to us reaching certainty about these truths. That is, until very recently the above mentioned causal explanations obtained reliability only from theoretical contexts. They were theoretical explanations, practically indisputable, but theoretical. And this raises the question whether the scientific explanation is not always -or almost always- dependent on the theory. If so, then the initial assumption that science provides causal explanations becomes problematic.

In Rivadulla (2004), I stated that "we use the expression theoretical explanation of a physical construct (explanandum) when we recover the construct in question by mathematical deduction under a more comprehensive law or theory, which serves as explanans. Thus any physical construct (fact, law, hypothesis) becomes theoretically explained when it reappears mathematically in the context of a broader physical construct. So not only facts but also empirical generalizations, abstract laws, and even theories themselves are susceptible to theoretical explanations in the sense indicated" (2004, p. 68). This version of scientific explanation deviates very slightly from Hempel's version, it releases specification of initial conditions, and thus it adjusts in a more precise form to the explanation processes in theoretical physics. Cases of theoretical explanations presented in Rivadulla (2004, pp. 71-84) speak for themselves.

\section{Hempel on scientific and causal explanations}

Hempel's D-N model of scientific explanation serves two purposes: 1."[to account] for a particular event by subsuming it under general laws" For instance, "the principles of Newtonian mechanics [...] explain certain 'general facts', i.e., empirical uniformities such as Kepler's laws of planetary motion; for the latter can be deduced from the former" (Hempel, 1965, pp. 173-174). And 2. "to explain the fact that a given law holds by showing that the latter is subsumable, in the same fashion, under more comprehensive laws or theoretical principles" (300) For example, "Newton's theory of gravitation can be subsumed, as an approximation, under that of the general theory of relativity" (300).

Scientific explanations are conceived, Hempel thinks, as "deductive arguments whose conclusion is the explanandum sentence, $E$, and whose premise-set, the explanans, consists of general laws, $L_{\gamma^{\prime}} L_{s^{\prime}} \ldots, L_{r}$ and other statements, $C_{\gamma^{\prime}} C_{s^{\prime}} \ldots, C_{k^{\prime}}$ which make assertions about particular facts" (Hempel, 1966, p. 51). These explanations also are called nomological-deductive explanations: "the explanatory information they provide implies the explanandum sentence deductively and thus offers logically conclusive grounds why the explanandum phenomenon is to be expected" (52). 
Hempel (1965, p. 174, 229) advances this model of explanation, but it is especially on p. 299 where he uses the expression "explanation by deductive subsumption under general laws, or briefly deductive-nomological explanation". And on p. $335 \mathrm{ff}$. he devotes a section labelled with this title. Now, not only facts, laws are also eligible for a scientific explanation according to the deductive covering law scheme: "the method of deductive-nomological explanation accounts for a particular event by subsuming it under general laws...; and it can similarly serve to explain the fact that a given law holds by showing that the latter is subsumable, in the same fashion, under more comprehensive laws or theoretical principles" (300). In this sense, Hempel argues both that the movement of the planets "exhibit the uniformities expressed by Kepler's laws [...] showing that these laws are but especial consequences of the Newtonian laws of gravitation and of motion" (1965, p. 343) and that the Newtonian theory of gravitation can be subsumed as an approach under the general theory of relativity. That the explanation should be extended to laws -that is, that the laws themselves should be taken as explicandum - is entirely reasonable and necessary in science. If the laws of Kepler are taken as very nearly true, then it must be possible to explain why they are, that is why they offer a reasonably good description of the movements of the planets. But I am not going to tackle in this paper the question of the scientific explanation of general laws.

The causal explanation of the solar eclipse, for instance, conforms to Hempel's D-N model:

- The general laws are: "In a certain strip on Earth darkness happens when the Moon intercepts the visual line between Sun and Earth."

- The initial conditions consist in the astronomical positions of Sun, Moon and Earth relative to each other viewed from a concrete fringe on Earth in a given time elapse.

Indeed, Hempel confirms: "the causal explanation implicitly claims that there are general laws -let us say, $L_{1}, L_{2}, \ldots, L_{r}$ in virtue of which the occurrence of the causal antecedents mentioned in $C_{1}, C_{2}, \ldots, C_{k}$ is a sufficient condition for the occurrence of the explanandum event. This relation between causal factors and effect is reflected in our schema (D-N): causal explanation is, at least implicitly, deductive-nomological" (1966, p. 349).

Well, if scientific explanation is approached from a logical point of view, then deductive subsumption is simply a purely logical approach to scientific explanation. The causal explanation, in case you might think that it is something other than a typical case of scientific explanation, also fits Hempel's nomologicaldeductive model (1965, p. 348). In the context of explanation, says Hempel, a cause is a complex set of circumstances and events described by a set of statements that correspond to the initial conditions of the nomological-deductive reasoning 
scheme. According to Hempel, causal explanations are special types of D-N explanation (300), and they conform to the D-N model (348). However, not every D-N explanation is a causal explanation (301). In particular, "the explanation of a general law by deductive subsumption under theoretical principles is clearly not an explanation by causes" (352). Briefly, all causal explanations are deductivenomological but not all D-N explanations are causal explanations.

\section{Einstein and Popper on causal explanation}

Karl Popper claims that "To give a causal explanation of an event means to deduce a statement which describes it, using as premises of the deduction one or more universal laws, together with certain singular statements, the initial conditions." Since, according to Popper, "It is from universal statements in conjunction with initial conditions that we deduce the singular statement" (1935, $\S 12$ ), it is obvious that Popper anticipates Hempel's D-N model of scientific explanation.

At least in his early writings, Popper identifies scientific explanation with causal explanation. For example, in his Postscript, he says that "it is the aim of science to find satisfactory explanations of whatever strikes us as being in need of explanation. By an explanation (or a causal explanation) is meant a set of statements one of which describes the state of affairs to be explained (the explicandum) while the others, the explanatory statements, form the 'explanation' in the narrower sense of the word (the explicans of the explicandum)" (1983, p. 132). However, in later writings identification between scientific explanation and causal explanation disappears. Thus, for example in (1972, Appendix § 6) Popper holds that an explanation is a logical deduction of the explicandum from certain premises (the explicans): general laws and singular initial conditions. This thesis already held Popper: "The scientist aims at finding a true theory or description of the World (and especially of its regularities or 'laws'), which shall also be an explanation of the observable facts. (This means that a description of these facts must be deducible from the theory in conjunction with certain statements, the socalled 'initial conditions')" (1963, p. 103).

No wonder that Popper $\left(1979, \S 11\right.$, note $\left.{ }^{*} 2\right)$ has ended realizing that his deductive conception of scientific explanation anticipates Hempel's deductivenomological model. This justifies that we should talk about the Popper-Hempel model of scientific explanation. Hempel mentioned only in passing (1965, p. 337, note 2) Section 12 of Popper's book, in which he anticipated his ideas.

In his 1927 article, Albert Einstein pointed out the following difference between Newton and Kepler from the point of view of the explanatory task of science: "Newton's object was to answer the question: is there any simple rule by 
which one can calculate the movements of the heavenly bodies in our planetary system completely, when the state of motion of all these bodies at one moment is known? Kepler's empirical laws of planetary motion, deduced from Tycho Brahe's observations, confronted him, and demanded explanation. These laws gave, it is true, a complete answer to the question of how the planets move round the sun: the elliptical shape of the orbit, the sweeping of equal areas by the radii in equal times, the relation between the major axes and the periods of revolution. But these rules do not satisfy the demand for causal explanation. They are logically independent rules, revealing no inner connection with each other".

This text brings to my mind two separate ideas. The first is that Kepler's laws of planetary motion are empirical and that they require an explanation, all physical laws that come directly from the experience need to be explained, as their genesis does not give the required explanation. Empirical or phenomenological laws of physics are not themselves explanatory. This is the case of Kepler's laws, the laws of falling bodies of Galileo, Boyle's Law, the Stefan's law of black body radiation, Wien's displacement law, or even the principles of classical or phenomenological thermodynamics. These laws are descriptive they tell which things happen and how they happen, but not why they do. In the case of Kepler's laws, it is what appears from the text of Einstein, they find the desired explanation in the context of Newtonian celestial mechanics. And in general this requirement of Einstein that empirical or phenomenological laws undergo an explanation evidences that the theorist is wary with the empirical or phenomenological beginning of science, which, as a theoretical construction must be deductive. Indeed, according to Einstein "as long as no principles are found on which to base the deduction, the individual empirical fact is of no use to the theorist; indeed he cannot even do anything with isolated general laws abstracted from experience. He will remain helpless in the face of separated results of empirical research, until principles which he can make the basis of deductive reasoning have revealed themselves to him" (1914, p. 221). Thus the theoretician is satisfied only when he has been able to derive the results -singular or general- from a given theoretical context. This weakens the argument that Kant presents in the first line of his Critique of Pure Reason, namely: "No one can doubt that all our knowledge begins with experience [...]. In time, therefore none of our knowledge precedes experience, and all begins in it".

The second idea that Einstein presents is that Kepler's laws, as they were originally presented, do not give any causal explanation of the planetary motions. They are three laws logically independent from each other. This seems reasonable, because if they require an explanation themselves, they could hardly offer a causal explanation of the events they describe. 


\section{Causal explanations. Are there any at all in theoretical science?}

In this section I focus on the explanation of Kepler's Third Law. My question is: can the deductive subsumption of Kepler's $3^{\text {rd }}$ Law (also known as Kepler's 1-2-3 law) under theoretical principles provide a causal explanation for the planetary motions?

My main concern in this section will be to clarify this issue in line with the answer to the following question: Is theoretical physics itself an explanatory science? From this question these other emerge: 1 . Does become an empirical law a causal law when it receives a scientific explanation? And 2. Are causal the theoretical laws of physics? The derivative question 1. has to do with the idea contained in the text of Einstein of 1927 that to the extent that the empirical laws of Kepler are explained by Newton, this explanation is causal. Is Einstein's position problematic? Question 2. requires a prior answer to the more general question, in order to clarify later on if scientific explanation is equivalent to causal explanation.

In any case, Hempel is sceptical about the existence of causal explanations: "Causal explanation in its various degrees of explicitness and precision is not, however, the only mode of explanation on which the D-N model has a bearing. For example, the explanation of a general law by deductive subsumption under theoretical principles is clearly not an explanation by causes. But even when used to account for individual events, D-N explanations are not always causal" (1966, p. 352). That is, not even for unique events the deductive-nomological explanations are always causal explanations, and of course the explanation of a law by another more general certainly is not. For instance, the theoretical explanation of Planck's radiation law in the framework of Bose-Einstein quantum statistics would not be causal. Thus, although all causal explanations are nomological-deductive not all nomological-deductive explanations are causal. And theoretical explanations of laws or theories by other broader or more general ones are not causal at all. Therefore Einstein's claim that Newton gives a causal explanation of Kepler is arguable! But why is it so?

Kepler's laws of planetary motions offer a very accurate description of what 'really' occurs. That is at least what is assumed in theoretical physics, where scientists' confidence in the third law -the square of the period of translation of any planet around the Sun is proportional to the cube of its mean distance from the sun $P^{2}=\left(\frac{4 \pi^{2}}{G_{N} M}\right) a^{3}-$ is out of question. This is also a much sought law in astrophysics and cosmology for its versatility and applicability in many circumstances, as I show in Rivadulla $(2015$, p. 111,173$)$. In this expression $P$ denotes the orbital period of a planet around the Sun (the same could be true for the period of any star, for example the Sun itself, around its galactic centre), $M$ is the mass of the Sun (or 
the total mass of a galaxy a star in the confines of the galaxy revolves around its centre), $G_{N}$ is Newton's gravitational constant, and a is the planet's distance from the Sun (in the case of circular orbit model we are using for simplicity reasons, a is the radius of the circular orbit of the planet, or otherwise the distance from the star to the galactic centre).

The derivation of this law in the framework of Newtonian mechanics, NM, in the more simple case of circular orbit is very elemental. For its deduction let simply be a binary system consisting of a body of mass $M$ and a body of mass $m$ separated by a distance $r(a$ in the formula above) among which a balance exists between attractive gravitational $F=G_{N} \frac{M m}{r^{2}}$ and centrifugal $F=m \times a_{n}=m \times \omega^{2} r=m \times\left(\frac{2 \pi}{P}\right)^{2} r$ forces. We express this balance as $G_{N} \frac{M}{r^{2}}=\frac{4 \pi^{2}}{P^{2}} r$, from which Kepler's formula derives.

So much this law is accepted that when in certain situations empirical data are opposed to it, the theoretical physicists risk to hypothesize the existence of, for example, dark matter to account for the observations, safeguarding the viability of the Kepler's third law (cf. Rivadulla, 2015, pp. 110-113). However, the reliability of Kepler's laws rests in the first instance on observation. For this reason, Einstein demanded that these laws should be explained themselves, and this is what Newton made. As a matter of fact, in the by the contemporary theoreticians considered relativistic 'Bible', Misner, Thorne and Wheeler express their admiration for the work of Newton: "No greater glory crowns Newton's theory of gravitation than the account it gives of the principal features of the solar system: a planet in its motion sweeps out equal areas in equal times; its orbit is an ellipse, with one focus at the sun; and the cube of the semimajor axis, a, of the ellipse multiplied by the square of the average angular velocity of the planet in its orbit $(\omega=2 \pi /$ period $)$ gives a number with the dimensions of a length, the same number for all the planets, equal to the mass of the sun: $M=\omega^{2} a^{3 \prime \prime}(1973$, p. 636). Kepler's third law is also called by Misner-Thorne-Wheeler 1-2-3 law due to the order of the exponents appearing in it.

There is no doubt, then, the explanation of Kepler's laws -and in particular the third law of planetary motion- takes place by mathematical deduction applicable in the context of Newtonian celestial mechanics. And it follows indeed PopperHempel's model of scientific explanation by deductive subsumption or, if you prefer, the more general model of theoretical explanation.

And now, we come to the key question: Provides Newton's derivation a causal explanation of Kepler's law and therefore a causal explanation of planetary motions? The answer to this question definitively depends on the response to the following issues: 1 . Is Newtonian mechanics the only theory able to offer a scientific explanation of Kepler's laws? 2. There is besides no other gravitational theory that could apply as an explanation of Kepler's laws? 
These are issues of the outmost importance, as any self-respecting theoretical physicist could not escape the fascinating task of trying to provide the theoretical explanation of Kepler's laws by subsumption under any other competent mature theory in gravitational affairs. Any other mature theory has to give an explanation of Kepler's laws!

Fortunately this theory exists and fulfills the task. It is, as we know, the general theory of relativity (GRT). Indeed, as M-T-W (1973) claim, Kepler's formula, Kepler's law for circular orbits, $M=\omega^{2} a^{3}$, obtains from the formula for the motion of a test particle in Swarzschild's geometry described by the line element (1973, 25.12, p. 655), (Exercise 25.19, p. 671). We find another deduction in N. Straumann $(2004, \S 3.6)$, among many others. Compared to the deduction of Kepler's law for circular orbits in the framework of Newtonian theory, this is like killing flies by gunfire. But in spite of being very complex mathematically the deduction of law 1-2-3 in the context of GRT does exist. GRT would have been stillborn if it were not able to allow within it the mathematical derivation of Kepler's Third Law!

In other words, instead of one, there are two scientific explanations of Kepler's laws. Is this good or bad? If we recall the words of Chang, with which we began this article, things seem not look good for physics. We have already seen that scientific explanation is not necessarily causal. But, it was unclear why. Moreover, if deducing an empirical law like 1-2-3 in a theoretical framework should be a causal explanation, then every derivation of it in a different theoretical context should also provide a causal explanation of the law. That is, if the Newtonian explanation of Kepler's law were causal, then the relativistic explanation should be causal too. And the same would happen for the theory of quantum gravity, or any alternative theory not yet proposed, if at some point it might become reality. This one would also be required to give a theoretical explanation of Kepler's Law. There can be no doubt about this.

The problem lies precisely in the causal principles assumed in every theory. For, if these principles were incompatible with each other then there would be as many causal explanations of the same phenomenon, or of the same law, as competing explanatory theories. The scientific and philosophical chaos would be complete! As a matter of fact, on occasion subsumptions occur under differing theoretical principles that are incompatible with one another. In such cases we would have incompatible scientific explanations of the same law. This is precisely the situation facing the scientific explanation of Kepler's laws, in particular the third law. Since there are incompatible gravitational theories, it is impossible for the scientific account of Kepler's law to be a causal explanation of the planetary motions. 
Indeed, the 'universal' laws that constitute the premises (explanans) of the deductive argument that is the scientific explanation of Kepler's third Law (explanandum) refer to efficient causes of totally different nature in the cases of NM and GRT: The existence of gravitational forces in the case of NM, and the curvature of spacetime in the case of GRT. The latter excludes the existence of gravitational forces acting at a distance and gives an explanation in terms of the geometry of spacetime. As M-T-W (1973, p. 649) claim: "Gravitation shows up in no way other than in curvature of the geometry, in which the particle moves as free of all 'real' force." Thus, both theories deny the fundamental postulates of each other. They are incompatible with each other.

Then, we face the following dilemma: either theoretical physics, like economics, as Ha-Joon Chang suspects, is not a science, because it allows the possibility of several incompatible explanations about the same phenomenon, or the concept of causal explanation in physics does not make sense. Since we can not afford to suspect that theoretical physics is not a science, then we must conclude that the concept of causal explanation is not viable in theoretical physics. If theoretical physics allows for the existence of several incompatible explanations of the same phenomenon, then the concept of causal explanation has no sense in it, unless one of the competing theories is wrong and the other true. But even in this case the problem is not trivial. For if we say that NM is false, that it has been conclusively refuted, this does not mean that we automatically accept that GRT is true. It will be, or will not, if it has the ability to be or not. But that we can never know. What would be clear is that Newton's third law explanation is not causal: The explanation provided by a wrong theory cannot be causal! And as a theoretical explanation it would be unsatisfactory. So Einstein's assumption that the Newtonian explanation of Kepler's law is a causal explanation of the planetary motions is highly arguable.

We could still complicate the situation if we turn to the theory of quantum gravity and the existence of such hypothetical entities called gravitons, and we would start over again.

\section{Conclusion}

Talking of causal explanations introduces of course a fascinating element in our expectations about science because it channels the scientific activity through the path of the search of the form of things themselves, of being able to get in touch with reality and to give a complete and accurate description of how and why the world is as it looks like. All of which is part of the efforts of the old metaphysics. 
But science, particularly theoretical physics, is more modest. It has no more pretensions than to put at our disposal tools -theoretical models and theories- to deal with, and intervene in, Nature with a success always moderate. It makes sense to dispense with the search for causal explanations in science.

In any case, and bearing in mind that when we make a not too problematic use of the term causal explanation there is always implied -more or less clearly-a reference to theory, then all scientific explanations -including causal explanationis ultimately a theoretical explanation. That is, all causal explanation is theoretical, but not all theoretical explanation is causal. I completely agree with Hempel that scientific explanations are not necessarily causal. Then we should renounce definitely to give causal explanations in theoretical physics.

Not wanting to draw the conclusion, that it would be exaggerated, that theoretical physics is not a science, it seems most reasonable to renounce in the field of scientific explanation to causal explanations -explanations by reference to efficient causes- in favour of the theoretical explanations -explanations in a given theoretical framework. Theoretical explanations are certainly less glamorous than causal explanations. And they also have an added cost: that we must renounce that all scientific explanations in principle are true, which means that, once accepted as such, they would be true once and for all and forever. But there is no other way out $\varphi$

\section{References}

Cartwright, N. (1989). Nature's Capacities and their Measurement. Oxford: Clarendon Press.

Einstein, A. (1914). Principles of Theoretical Physics. Inaugural address before the Prussian Academy of Sciences. Reprinted in A. Einstein. (1973). Ideas and Opinions, (221-223). London: Souvenir Press Ltd.

Einstein, A. (1927). The Mechanics of Newton and their Influence on the Development of Theoretical Physics. Die Naturwissenschaften, 15 (1927). Reprinted in A. Einstein. (1973). Ideas and Opinions, (221-223). London: Souvenir Press Ltd.

Hempel, C. G. (1965). Aspects of Scientific Explanation and Other Essays in the Philosophy of Science. New York: Free Press.

Hempel, C. G. (1966). Philosophy of Natural Science. Englewood Cliffs, N. J: Prentice-Hall, Inc. 
Misner, Ch.; Thorne, K. \& Wheeler, J. A. (1973). Gravitation. New York: Freeman and Company.

Popper, K. (1959). The Logic of Scientific Discovery. London: Hutchinson.

Popper, K. (1963). Conjectures and Refutations. The Growth of Scientific Knowledge. London: Routledge and Kegan Paul.

Popper, K. (1972). Objective Knowledge. Oxford: Clarendon Press.

Popper, K. (1979). Die beiden Grundprobleme der Erkenntnistheorie. Tübingen: J. C. B. Mohr (Paul Siebeck).

Popper, K. (1983). Realism and the Aim of Science. London: Hutchinson.

Rivadulla, A. (1991). Probabilidad e Inferencia Científica. Barcelona: Anthropos.

Rivadulla, A. (2004). Éxito, Razón y Cambio en Física. Madrid: Trotta.

Rivadulla, A. (2015). Meta, Método y Mito en Ciencia. Madrid: Trotta.

Straumann, N. (2004). General Relativity. With Applications to Astrophysics. Berlin: Springer. 